\title{
Aplicação de Grounded Theory para Investigar Iniciativas de Implementação de Melhorias em Processos de Software
}

\author{
Mariano Angel Montoni, Ana Regina Cavalcanti da Rocha \\ COPPE/UFRJ - Universidade Federal do Rio de Janeiro \\ Caixa Postal 68511 - CEP 21945-970 - Rio de Janeiro - RJ - Brasil \\ \{mmontoni, darocha\}@cos.ufrj.br
}

\begin{abstract}
Resumo. Estudos recentes apresentam relatos das dificuldades que as organizações enfrentam para implementar melhorias em processos de software com base em modelos e padrões de processos, principalmente relacionadas à incapacidade de superar barreiras críticas como falta de motivação e apoio da alta gerência. O entendimento adequado do contexto sociocultural no qual são conduzidas melhorias em processos de software pode facilitar o desenvolvimento de estratégias de implementação de melhorias em processos mais eficientes para superar tais dificuldades. $O$ objetivo deste trabalho é apresentar um estudo baseado em Grounded Theory conduzido para investigar iniciativas de implementação de melhorias em processos de software na perspectiva de consultores de organizações de consultoria. O produto principal deste estudo é uma teoria construída com o propósito de explicar o fenômeno associado ao sucesso de iniciativas de implementação de melhorias em processos de software.
\end{abstract}

\begin{abstract}
Recent studies show that many organizations struggle to implement Software Process Improvement (SPI) based on process models and standards mainly because of incapacity to overcome critical barriers, such as lack of motivation and higher management support. The accurate understanding of the social-cultural context where SPI is undertaken can facilitate the development of more efficient SPI implementation strategies aiming to overcome those difficulties. The objective of this work is to present a Grounded Theory based study conducted to investigate SPI implementation initiatives in the perspective of consultants of SPI consultancy organizations. The main product of this study is a theory that tries and explains the phenomenon associated to the success of SPI implementation initiatives.
\end{abstract}

\section{Introdução}

Iniciativas de implementação de melhorias em processos de software têm sido largamente conduzidas em organizações de software com o propósito de aderir a 'melhores práticas' preconizadas por modelos e padrões de referência de processos. No entanto, estudos recentes apresentam relatos das dificuldades que as organizações enfrentam para implementar melhorias em processos de software com base em modelos e padrões de processos, principalmente relacionadas à incapacidade de superar barreiras críticas como falta de motivação e apoio da alta gerência (Baddoo e Hall, 2003; Staples et al., 2007). O entendimento adequado do contexto sociocultural no qual são conduzidas melhorias em processos de software pode facilitar o desenvolvimento de 
estratégias de implementação de melhorias em processos mais eficientes para superar tais dificuldades.

O objetivo deste trabalho é apresentar um estudo conduzido para investigar iniciativas de implementação de melhorias em processos de software na perspectiva de consultores de organizações de consultoria. A metodologia escolhida para guiar o estudo é Grounded Theory pois é indicada para estudar o comportamento humano e culturas organizacionais (Strauss e Corbin, 1998; Coleman e O'connor, 2007). Outras razões que subsidiam a escolha da Grounded Theory para guiar o estudo estão relacionadas à facilidade em gerar conhecimento de forma indutiva a partir das experiências de implementadores de melhorias em processos, além de ser uma metodologia estabelecida e acreditada em áreas de ciências sociais e saúde (Strauss e Corbin, 1998).

A próxima seção apresenta alguns estudos da aplicação de Grounded Theory nas áreas de engenharia de software e melhoria de processos. A seção 3 apresenta a metodologia de pesquisa. A aplicação da Grounded Theory para guiar o estudo é apresentada na seção 4. A seção 5 apresenta os resultados do estudo. Finalmente, as conclusões do trabalho e os próximos passos são apresentados na seção 6 .

\section{Aplicação da Grounded Theory em Pesquisas Qualitativas em Engenharia de Software e Melhoria de Processos}

Apesar da Ground Theory ter sido originada nos anos 60 e ter sido extensivamente aplicada nas áreas de ciências sociais; o uso da Grounded Theory em estudos na área de desenvolvimento de software não é muito comum, sendo geralmente restrita para investigar questões tecnológicas na área de Sistemas de Informação. A aplicação da Grounded Theory nas áreas de engenharia de software e melhoria de processo é ainda mais escassa. No entanto, existem alguns estudos relevantes que devem ser destacados.

Um estudo interessante descrevendo a aplicação da Grounded Theory é apresentado por ORLIKOWSKI (Orlikowski, 1993). Neste trabalho, o autor caracteriza a experiência de duas organizações com a adoção e uso de ferramentas CASE. Alguns dos resultados desse estudo sugerem que a implementação de ferramentas CASE pode ser mais bem gerenciada se os implementadores reconhecem que a implementação de ferramentas CASE não é meramente uma instalação tecnológica, mas envolve um processo de mudança organizacional ao longo do tempo. Apesar desse trabalho não focar especificamente em melhorias em processos de software, os resultados do estudo têm importantes implicações para a pesquisa e prática de melhorias em processos de software, pois a implementação de ferramentas CASE pode ser interpretada como uma variação de melhoria de processo. Esse trabalho enfatiza a adequação da metodologia de Grounded Theory para investigar o contexto social que permeia a introdução de melhorias em processos de software, porém os resultados apresentados não fornecem conhecimento prático para superar barreiras para implementar com sucesso melhorias em processos de software.

Outro trabalho na área de melhoria em processos de software descrevendo resultados interessantes com a adoção de Grounded Theory é apresentada por COLEMAN e O'CONNOR (Coleman e O'connor, 2008). Os autores investigaram a prática de processo de software na indústria irlandesa de software e concluíram que programas de melhorias em processos de software são implementados de forma reativa e que existe 
alguma resistência por parte dos gerentes de software para implementar modelos de melhores práticas para melhoria de processos de software devido aos custos associados. Apesar dos resultados desse estudo terem implicações significativas na área de melhoria de processos de software, esses resultados são dependentes de contexto e, conseqüentemente, tem utilidade limitada em diferentes cenários.

\section{Metodologia de Pesquisa}

Para apoiar a aplicação da metodologia de Grounded Theory, foi desenvolvido um procedimento baseado nas descrições dos componentes da Grounded Theory, apresentados em (Strauss e Corbin, 1998; Bandeira-De-Mello e Cunha, 2003; Carvalho et al., 2003). A Figura 1 apresenta o esquema geral da metodologia de pesquisa. Em seguida, são descritos os passos do procedimento adotado para guiar a pesquisa.

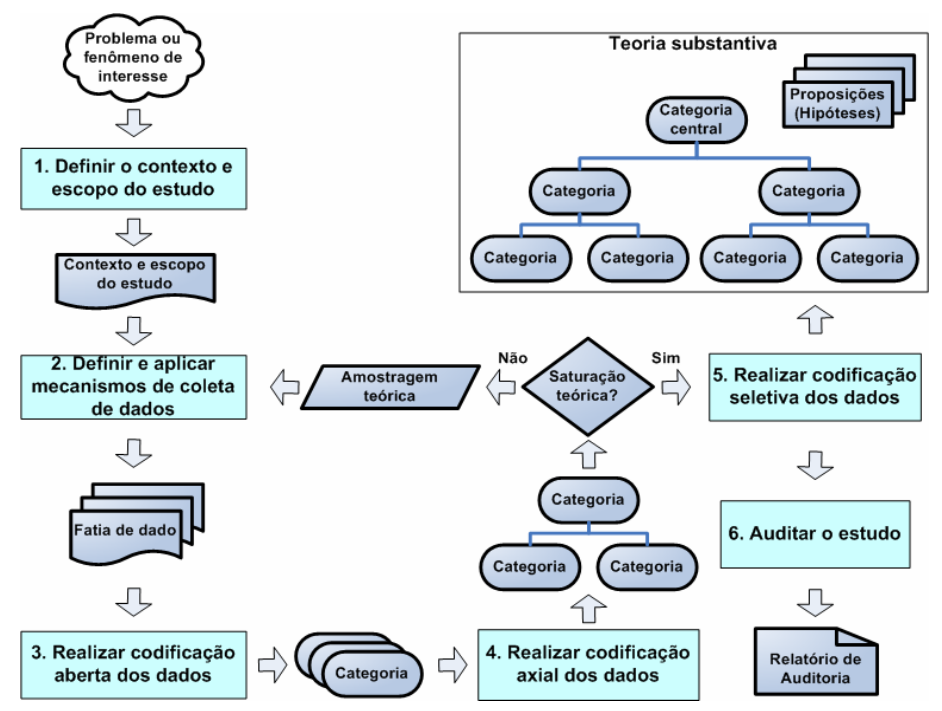

Figura 1. Esquema geral da metodologia de pesquisa.

\section{$1^{\circ}$ Passo - Definir o contexto e escopo do estudo}

Inicialmente, devem ser definidos o contexto e o escopo para estudo de um fenômeno. A base da Grounded Theory é que uma teoria é desenvolvida de forma indutiva a partir dos dados, sendo gerada (ou fundamentada) em um processo contínuo de amostragem e análise de dados. A capacidade de generalização da teoria construída é dependente do quanto o contexto e escopo definidos representam o fenômeno estudado (Strauss e Corbin, 1998). Quanto maior for a abrangência e generalidade dos dados coletados no estudo, maior será a generalização da teoria construída. Portanto, múltiplas fontes de dados devem ser utilizadas para aumentar a confiabilidade dos resultados e facilitar a captura do conhecimento, visão e perspectiva dos envolvidos no estudo sobre um aspecto do fenômeno sob investigação (Bandeira-De-Mello e Cunha, 2003; Carvalho et al., 2003).

\section{$2^{\circ}$ Passo - Definir e aplicar mecanismos de coleta de dados}

Este passo envolve a definição e aplicação de mecanismos de coleta de dados. Os mecanismos apontados como sendo os mais comuns e aplicáveis em estudos de análise qualitativos baseados em Grounded Theory são: (i) estudos experimentais do tipo 
survey, (ii) revisão da literatura e (iii) entrevistas semi-estruturadas (Strauss e Corbin, 1998; Carvalho et al., 2003). A aplicação desses mecanismos permite criar um repositório de dados (ou fatias de dados no jargão da Grounded Theory) que servirá de base para investigação de um determinado fenômeno. A utilização de ferramentas de apoio é importante para facilitar o armazenamento desses dados, bem como a sua recuperação e análise. Uma ferramenta bastante adotada em estudos qualitativos com base na Grounded Theory é a ferramenta ATLAS.ti ${ }^{1}$.

\section{$3^{0}$ Passo - Realizar codificação aberta dos dados}

Após a coleta dos dados, a análise pode ser iniciada. A relação dinâmica entre a análise e a coleta de dados é uma característica significativa da Grounded Theory (Carvalho et al., 2003). A análise de dados orientada pela Grounded Theory é realizada por meio da codificação dos dados. São previstos três tipos de codificação: codificação aberta, axial e seletiva (Strauss e Corbin, 1998; Bandeira-De-Mello e Cunha, 2003). A codificação aberta ou rotulação (labeling) envolve a quebra, análise, comparação, conceituação e categorização dos dados. A codificação axial examina as relações entre as categorias e subcategorias dos dados. Já a codificação seletiva refina todo o processo, identificando a categoria central da teoria, com a qual todas as outras estão relacionadas.

Os objetivos deste passo são os seguintes: (i) realizar a codificação aberta dos dados coletados e (ii) agrupar os códigos em categorias e subcategorias. Neste contexto, a codificação compreende o processo de gerar rótulos (ou códigos) para descrever conceitos e aspectos relevantes de certas passagens de textos nas fontes dos dados (Seaman, 1999). A análise dos dados é realizada por meio da busca por similaridades e diversidades entre os dados, coletando um número de indicadores que apontem para múltiplos aspectos qualitativos de um conceito potencialmente significativo.

\section{$4^{\circ}$ Passo - Realizar codificação axial dos dados}

O objetivo deste passo é agregar conhecimento sobre a teoria. Na Grounded Theory, o processo investigativo de construção de uma teoria é guiado por um modelo chamado Modelo de Paradigma (Strauss e Corbin, 1998). Segundo esse modelo, conhecimento é agregado a uma teoria por meio da análise e construção das relações entre categorias e subcategorias que devem ser testadas novamente nos dados. A Grounded Theory denomina esse tipo de análise de codificação axial. As relações entre as categorias e subcategorias da teoria são definidas na forma de proposições (ou hipóteses), representando condições causais, fenômeno, contexto, condições intervenientes, estratégias de ação/interação e conseqüências. A construção de uma teoria deve ser guiada pela busca contínua de conexões entre instâncias desses elementos, representados na forma de proposições ou hipóteses fundamentadas nos dados coletados.

\section{$5^{\circ}$ Passo - Realizar codificação seletiva dos dados}

O objetivo deste passo é refinar o conhecimento sobre a teoria construída por meio do que a Grounded Theory denomina de codificação seletiva. Neste passo, todo o processo

\footnotetext{
${ }^{1}$ ATLAS.ti é uma ferramenta desenvolvida pela Scientific Software Development Gmbh para apoiar a análise visual de dados qualitativos. Esta ferramenta foi utilizada no estudo apresentado neste trabalho.
} 
de construção da teoria é refinado por meio da identificação da categoria central da teoria. A categoria central deve ser capaz de integrar todas as outras categorias e expressar a essência do processo social que ocorre entre os envolvidos (Bandeira-DeMello e Cunha, 2003). Categorias mal formuladas devem ser revistas neste passo, bem como devem ser resolvidas falhas na lógica da teoria.

A construção de uma teoria fundamentada em dados deve ser realizada de forma iterativa à medida que os dados são coletados. Em cada iteração do processo de pesquisa, mais conhecimento sobre a teoria é agregado e refinado até que a coleta e análise de mais dados não proporcionem ganhos significativos à teoria, ou seja, novas categorias e relações não sejam mais encontrados no processo de codificação, alcançando a saturação teórica (Strauss e Corbin, 1998). Caso não tenha sido alcançada a saturação teórica ao final de uma iteração do processo de pesquisa, uma nova amostragem teórica de dados deve ser realizada seguida de mais uma iteração de coleta e análise de dados.

\section{$6^{0}$ Passo - Auditar o estudo}

A técnica Auditorias deve ser aplicada em todo o processo de investigação para garantir validade dos resultados encontrados. Para tanto, é fundamental que sejam registradas anotações ao longo de todo o processo, desde a etapa de coleta dos dados até a codificação e análise dos elementos construtores da teoria. A aplicação da técnica Auditorias deve ser guiada por um conjunto de critérios definidos e aplicada por uma pessoa que não esteja envolvida no processo de pesquisa para garantir objetividade dessa avaliação (Strauss e Corbin, 1998). Exemplos de critérios para serem utilizados na avaliação da teoria são: grau de coerência, relevância, flexibilidade e densidade (Bandeira-De-Mello e Cunha, 2003).

\section{O Estudo baseado em Grounded Theory}

Para focar a aplicação da metodologia de Grounded Theory, as seguintes questões de pesquisa foram investigadas em duas fases distintas do estudo: (QP1) Quais são os fatores que influenciam o sucesso de iniciativas de melhoria em processos de software? e (QP2) Quais são as estratégias mais adequadas para implementar melhoria em processos de software em determinados contextos?. A Figura 2 apresenta a estrutura geral do estudo baseado em Grounded Theory. A aplicação da metodologia nas duas fases do estudo para investigar as questões de pesquisa é apresentada em seguida.

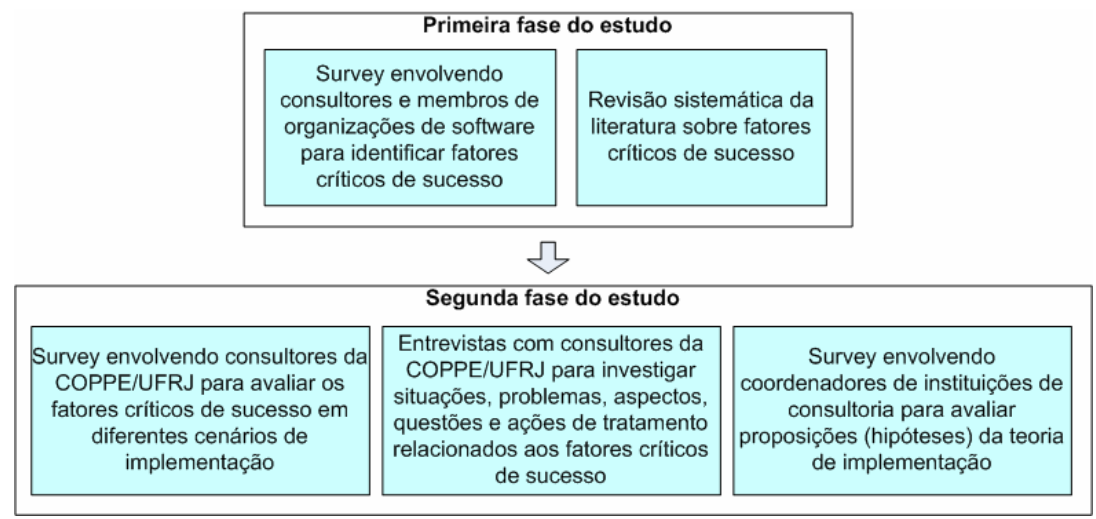

Figura 2. Estrutura geral do estudo baseado em Grounded Theory. 


\subsection{Primeira Fase do Estudo}

A primeira fase do estudo foi restringida para analisar apenas experiências de um grupo específico de consultores e membros de organizações de software envolvidas em iniciativas de melhoria. A aplicação detalhada, nesta primeira fase do estudo, da metodologia de Grounded Theory é apresentada em (Montoni e Rocha, 2007) e descrita brevemente a seguir.

Os participantes da primeira fase do estudo foram selecionados a partir de um grupo de organizações de consultoria com experiências bem sucedidas de implementação de melhorias em processos de software baseada no modelo CMMI (Chrissis et al., 2006) e MR-MPS (Softex, 2009). Também foram selecionados membros das organizações de software que foram alvo das iniciativas de melhoria coordenadas por essas organizações de consultoria.

Os dados foram coletados por meio da aplicação de dois tipos de questionários, um questionário foi submetido para consultores de melhoria em processos de software e o outro para membros de organizações de software que conduziram melhorias nos seus processos. Basicamente, os dois questionários continham as mesmas questões variando apenas as informações de caracterização do participante do estudo. As questões dos questionários eram duas perguntas abertas, uma sobre os fatores que dificultam a implantação de processos de software e outra sobre os fatores que facilitam a implantação de processos de software.

No total, foram retornados 25 questionários contendo descrições gerais sobre fatores que influenciam iniciativas de melhorias em processos de software. Desse conjunto, 17 questionários eram de implementadores de 5 diferentes organizações e 8 questionários de membros representando 5 diferentes organizações de software. Devido ao pequeno número de questionários retornados pelos membros das organizações de software, optou-se por não distinguir os dois subconjuntos nos procedimentos de análise. Porém, pode ser importante separar as análises entre esses dois grupos em estudos futuros para entender sob diferentes perspectivas o fenômeno da implementação de melhorias em processos de software.

Os questionários foram analisados procurando classificar cada passagem de texto em uma categoria representando um tipo de achado de um fator capaz de influenciar tanto de forma positiva quanto negativa o sucesso de iniciativas de melhorias em processos de software. A partir da análise dos 25 questionários, foi possível identificar 66 diferentes categorias (códigos) de tipos de achados de fatores críticos de sucesso.

Devido ao caráter exploratório desta primeira fase do estudo, também se considerou relevante analisar resultados reportados por outros estudos experimentais na área de melhoria de processos de software com o propósito de identificar e analisar os fatores que exercem influência sobre as iniciativas de melhoria. A partir da execução desse estudo, foram selecionados 10 estudos experimentais considerados relevantes. Foi considerado como um fator crítico, apenas aqueles identificados por mais de $40 \%$ dos estudos. Este conjunto de fatores críticos foi então analisado procurando associar cada fator apontado nos estudos a um dos 66 códigos de tipos de fatores críticos de sucesso previamente identificados. No entanto, não foi possível associar todos os fatores a um desses códigos. Para esses casos, foram criados 10 novos códigos. No total, foram identificados 25 códigos de propriedades relacionadas a 12 fatores críticos de sucesso. 
O conhecimento teórico foi agregado por meio da codificação axial dos dados o que implicou realizar análise e construção das relações entre os códigos identificados. Como não havia conhecimento prévio sobre fatores críticos de sucesso, procurou-se superar essas dificuldades identificando relações entre os códigos por meio da aplicação de análise estatística de dados capaz de apoiar a construção de relações estatisticamente significativas entre os dados coletados. Neste contexto, foram aplicadas as técnicas de análise estatística denominadas MDS e PCA com o propósito de derivar e agregar componentes principais de fatores críticos de sucesso. Foi denominado de "Componente de Fator Crítico de Sucesso" o conjunto de categorias de propriedades de fatores críticos de sucesso com relações estatisticamente significativas. Esses componentes foram analisados e relacionados a uma categoria central denominada "Sucesso de Iniciativa de Melhoria" como o resultado do processo iterativo de refinamento e integração dos dados. No total, foram identificados 5 componentes. A Tabela 1 apresenta esses componentes e as propriedades de fatores críticos de sucesso com relacionamento estatisticamente significativo.

Tabela 1. Componentes de fatores críticos de sucesso

\begin{tabular}{|l|l|}
\hline$\#$ & \multicolumn{1}{|c|}{ Propriedades de Fatores Críticos de Sucesso } \\
\hline 1 & $\begin{array}{l}\text { Competências em engenharia de software dos membros da organização } \\
\text { (conhecimento, experiências e habilidades) } \\
\text { Inexistência de conflitos de interesses na implementação de processos } \\
\text { Estabilidade interna na organização } \\
\text { Satisfação dos membros da organização } \\
\text { Existência de política de reconhecimento à colaboração na melhoria dos processos } \\
\text { Relacionamento dos membros da organização com a consultoria especializada }\end{array}$ \\
\hline 2 & $\begin{array}{l}\text { Conscientização dos membros da organização quanto aos benefícios obtidos com } \\
\text { a implantação dos processos } \\
\text { Existir gerência do projeto de implementação da melhoria dos processos }\end{array}$ \\
\hline 3 & $\begin{array}{l}\text { Balanceamento entre as melhorias impostas pela alta direção, as necessidades do } \\
\text { modelo adotado e as melhorias propostas pelos membros da organização } \\
\text { Rotatividade de pessoal da organização } \\
\text { Adequação dos processos/procedimentos definidos } \\
\text { Completa institucionalização das melhorias implementadas nos projetos }\end{array}$ \\
\hline 4 & $\begin{array}{l}\text { Apoio efetivo da alta gerência } \\
\text { Competências em engenharia de software dos membros da organização } \\
\text { (conhecimento, experiências e habilidades) } \\
\text { Disponibilidade de recursos financeiros da organização para atividades de } \\
\text { melhoria de processo } \\
\text { Disponibilidade de tempo dos membros da organização para atividades de } \\
\text { melhoria de processo }\end{array}$ \\
\hline 5 & $\begin{array}{l}\text { Facilidade de aceitação de mudanças } \\
\text { Motivação dos membros da organização } \\
\text { Confiança dos membros da organização na consultoria especializada }\end{array}$ \\
\hline
\end{tabular}

O fator 1 é rotulado de "Ambiente" pois todas as variáveis medem a capacidade ambiental para estabelecer e manter iniciativas de melhoria de processo de software. $\mathrm{O}$ fator 2 é rotulado de "Estratégia" e indica que uma estratégia eficiente de melhoria de 
processo de software deve garantir que os membros da organização têm consciência dos benefícios potenciais que podem ser alcançados com a implementação da melhoria de processo de software. O fator 3 foi nomeado de "Institucionalização" pois as variáveis desse fator medem o grau de institucionalização das melhorias implementadas através da organização pela caracterização do grau de resistência da institucionalização dos processos e procedimentos a mudanças estruturais da organização. Como todas as variáveis do fator 4 são consideradas indicadores de comprometimento para a melhoria de processo de software, esse fator foi rotulado de "Comprometimento". O fator 5 é rotulado de "Motivação e aceitação" e indica que a equipe de melhoria de processo de software é um facilitador da aceitação dos membros da organização para a institucionalização de mudanças nos processos promovidas pelas iniciativas de melhoria de processo de software.

\subsection{Segunda Fase do Estudo}

A segunda fase do estudo foi restringida para analisar experiências de um grupo de implementadores de uma instituição específica de consultoria de melhoria em processos de software. A instituição selecionada para este estudo foi a COPPE/UFRJ, devido à facilidade de acesso aos seus implementadores e ao grande número de experiências passadas de iniciativas de melhoria de sucesso coordenadas por essa instituição. $\mathrm{O}$ escopo da segunda fase do estudo se limitou a analisar apenas as iniciativas de melhoria baseadas nos modelos CMMI e MR-MPS e que tiveram avaliação oficial bem sucedida em algum desses modelos.

Inicialmente, procurou-se avaliar a criticidade dos fatores identificados na primeira fase do estudo em diferentes contextos de implementação. Para tanto, foi executado um survey envolvendo os consultores da COPPE/UFRJ com o propósito de identificar os fatores mais críticos de sucesso em cenários específicos de implementação de melhorias em processos de software. Foram identificadas as informações das iniciativas passadas coordenadas pela COPPE/UFRJ. Em seguida, cada iniciativa foi categorizada em um cenário específico de acordo com o nível de maturidade da unidade organizacional alvo das atividades de implementação e o nível de maturidade pretendido. Os implementadores envolvidos em cada iniciativa também foram identificados, bem como o papel exercido por cada um deles no contexto de cada iniciativa. Os papéis identificados foram os seguintes: Coordenador da II (Instituição Implementadora), Gerência da equipe de implementação, Definição de processos e Equipe de implementação. No total, foram identificadas 30 iniciativas de melhoria, categorizadas em 6 diferentes cenários de implementação, envolvendo 16 implementadores. Os cenários identificados foram os seguintes: (i) Iniciando a implementação pelo nível $G$ do MPS, (ii) Iniciando a implementação pelo nível F do MPS ou 2 do CMMI, (iii) Iniciando a implementação pelo nível E do MPS, (iv) Implementando o nível E do MPS em uma organização com nível G do MPS, (v) Implementando o nível E do MPS em uma organização com nível F do MPS ou 2 do CMMI e (vi) Implementando o nível C do MPS ou 3 do CMMI em uma organização com nível F do MPS ou 2 do CMMI.

Foi definido um questionário para apoiar a realização do survey com os implementadores da COPPE/UFRJ. O questionário continha uma matriz relacionando os fatores críticos de sucesso (medidos pelas suas propriedades extraídas da teoria construída na primeira fase do estudo) e os cenários de implementação que o 
participante do estudo esteve envolvido de forma significativa. O questionário foi enviado para cada um dos 16 implementadores envolvidos nas iniciativas coordenadas pela COPPE/UFRJ. Todos os implementadores retornaram o questionário respondido. Os questionários retornados foram consolidados de acordo com o perfil de cada participante em cada um dos cenários de implementação. O perfil dos participantes foi definido com base nos papéis exercidos nas iniciativas de melhoria enquadradas em cada cenário de implementação.

Alguns participantes do survey identificaram outros fatores, além daqueles apresentados no questionário. Os passos da metodologia executados na primeira fase do estudo foram novamente executados com base nesses novos dados. Com base no dicionário de fatores críticos de sucesso extraído da primeira fase do estudo, foram associados códigos de tipos de achado aos outros fatores identificados pelos participantes do survey. No entanto, nem todos os fatores puderam ser associados aos códigos existentes. Para esses casos, 13 novos códigos foram identificados e incluídos no dicionário de dados. Também foi necessário estender o conhecimento teórico por meio da criação de 4 novos códigos de propriedades de fatores críticos de sucesso.

Os resultados das respostas dos perfis dos participantes foram consolidados considerando os diversos cenários de implementação. Cada fator foi analisado procurando identificar os cenários em que o fator foi considerado "Muito Crítico" ou "Crítico" para cada um dos grupos de implementadores. O resultado do survey foi que todos os fatores foram avaliados como "Muito Crítico" ou "Crítico" por todos os grupos de participantes do estudo em um ou mais cenários. Portanto, pode-se verificar que todos os fatores identificados na primeira fase do estudo são de fato críticos no contexto atual de investigação. Além disso, foi possível identificar novos fatores considerados críticos pelos participantes do estudo e que também devem ser investigados nesta fase do estudo.

Após a avaliação da criticidade dos fatores em diferentes contextos de implementação, foi planejada e conduzida uma série de entrevistas com os consultores da COPPE/UFRJ com o propósito de investigar sobre as situações, problemas, aspectos ou questões capazes de influenciar tanto de forma positiva quanto negativa a presença de fatores críticos de sucesso.

No total, foram entrevistados 6 consultores experientes em três diferentes grupos de acordo com o perfil (1 coordenador da II, 2 gerentes de equipes de implementação e 3 membros de equipes de implementação). As entrevistas também tiveram como propósito investigar como uma organização de consultoria pode ajudar a garantir e maximizar a influência positiva, bem como a eliminar ou minimizar a influência negativa dos fatores críticos de sucesso.

Um questionário foi desenvolvido para apoiar a condução das entrevistas. Esse questionário foi preenchido ao longo da condução das entrevistas para facilitar tanto o registro das informações de forma coletiva pelos entrevistados, quanto a posterior análise dos dados coletados. Basicamente, o questionário continha 29 seções, uma para cada fator crítico de sucesso. Em cada uma dessas seções foram apresentadas 2 tabelas para registrar as situações, problemas, aspectos ou questões capazes de influenciar a presença de cada fator. Na primeira tabela foram registradas as influências negativas, enquanto na segunda tabela foram registradas as influências positivas dos fatores 
analisados. As tabelas continham também áreas específicas para identificar as ações que podem ser realizadas para ajudar a eliminar ou minimizar a influência negativa, bem como para ajudar a garantir ou maximizar a influência positiva do fator, além de áreas específicas para identificar as conseqüências esperadas dessas ações ou da falta delas.

Em seguida, foi realizada a análise iterativa de codificação aberta e axial dos dados das entrevistas. Como resultado dessa análise, foi elaborado um conjunto de proposições (hipóteses) associadas a cada um dos fatores críticos de sucesso. Essas proposições foram avaliadas pelos entrevistados que sugeriram modificações, quando apropriado. Após a avaliação das proposições, foi realizada a análise seletiva dos dados com o propósito de identificar as categorias principais da teoria em construção.

O estágio atual do estudo envolve a avaliação das proposições (hipóteses) por um grupo de coordenadores de instituições de consultoria em melhorias de processos de software. A partir da análise do resultado dessa avaliação será possível obter uma visão mais acurada do fenômeno de implementação de melhorias em processos de software considerando as experiências de diferentes organizações de consultoria.

\section{Resultados do Estudo}

A metodologia de Grounded Theory apoia o desenvolvimento de teorias de forma sistemática por meio da coleta e análise de dados originados nas experiências dos indivíduos. Uma teoria pode ser definida como "um conjunto de categorias (conceitos) sistematicamente inter-relacionadas por meio de sentenças de relacionamento para formar um framework teórico capaz de explicar um fenômeno social relevante" (Strauss e Corbin, 1998). Como resultado do estudo, foram identificados dois conjuntos de categorias centrais denominadas de "Contexto Institucional para Implementação de Melhorias em Processos de Software" e "Comportamento Estratégico na Implementação de Melhorias em Processos de Software" representando, respectivamente, as características de contexto que podem influenciar o sucesso de uma iniciativa de melhoria e as ações estratégicas realizadas por atores de uma iniciativa de melhoria para reforçar ou modificar tais influências. A Figura 3 apresenta as categorias relacionadas às categorias centrais que formam o framework teórico.

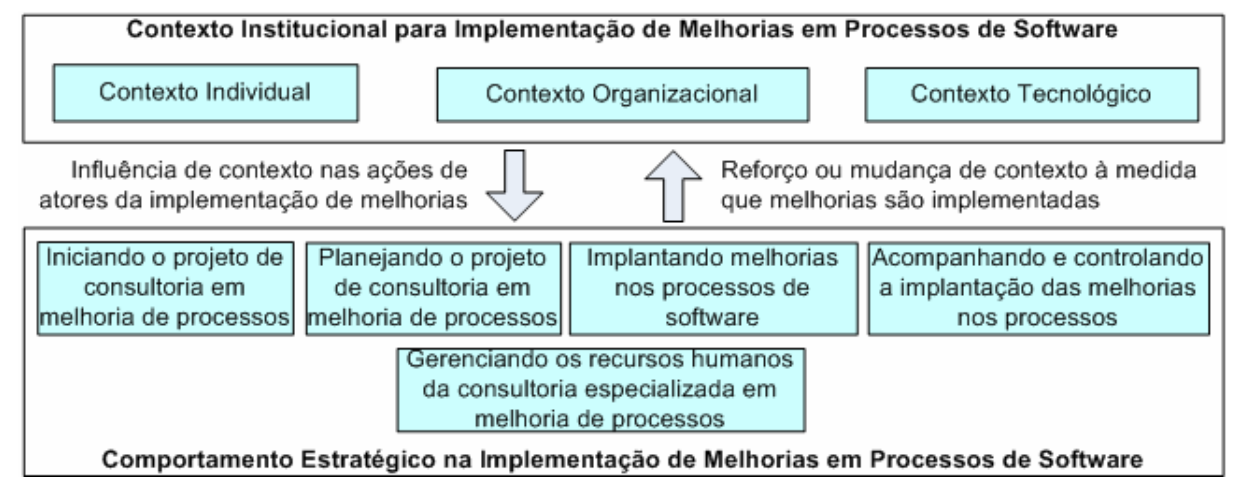

Figura 3. Processo de mudança organizacional para ter sucesso na condução de iniciativas de implementação de melhorias em processos de software.

Três tipos de categorias de contexto foram identificados. Esses contextos exercem diferentes influências no sucesso de iniciativas de melhoria. Os atores dessas iniciativas reagem a essas influências por meio da tomada de diferentes ações categorizadas em 5 
grupos distintos. Esse framework teórico é apresentado em detalhes a seguir juntamente com exemplos de proposições extraídas da teoria para apoiar os achados.

\section{Contexto Institucional para Implementação de Melhorias em Processos de Software}

A primeira categoria central da teoria agrega as características de contexto em três categorias. Os conceitos de cada uma dessas categorias são apresentados na Tabela 2 e suas propriedades são descritas em seguida.

Tabela 2. Conceitos relacionados às categorias de contexto

\begin{tabular}{|l|l|}
\hline \multicolumn{1}{|c|}{ Categoria } & \multicolumn{1}{c|}{ Conceito } \\
\hline Contexto Individual & $\begin{array}{l}\text { Membros da organização } \\
\text { Alta gerência } \\
\text { Membros da consultoria especializada em melhoria de } \\
\text { processos }\end{array}$ \\
\hline \multirow{3}{*}{ Contexto Organizacional } & $\begin{array}{l}\text { Estratégias e políticas organizacionais } \\
\text { Programa de Melhoria de Processos } \\
\text { Ambiente }\end{array}$ \\
\hline Contexto Tecnológico & $\begin{array}{l}\text { Processos de software } \\
\text { Recursos de software e hardware de apoio aos processos } \\
\text { de software }\end{array}$ \\
\hline
\end{tabular}

- Membros da organização: Este é o conceito de contexto com maior densidade sendo constituído de 17 propriedades variando desde o comprometimento e envolvimento dos membros da organização até a facilidade de aceitação de mudanças. Este fato mostra que um dos aspectos mais críticos para ter sucesso na condução de iniciativas de melhoria reside nas reações das pessoas que irão executar as melhorias na organização e nos projetos.

- Alta gerência: Este conceito é constituído de duas propriedades indicando a efetividade do apoio na implementação das melhorias e a confiança da alta gerência na coordenação da consultoria. Apesar deste conceito ter baixa densidade, possui influência significativa em outras propriedades. Segundo o coordenador da instituição de consultoria "quando existe apoio e cobrança da alta gerência é mais provável ter-se facilidade de aceitação de mudanças".

- Membros da consultoria especializada em melhoria de processos: Este conceito mostra que características dos membros da consultoria, como adequação das competências, perfil e flexibilidade, exercem influências em outras propriedades como no relacionamento e confiança dos membros da organização e no alinhamento adequado da implementação dos processos com as especificidades da empresa. Segundo o coordenador da instituição de consultoria "quando falta à consultoria experiência e maturidade profissional para lidar com situações complicadas, é provável que este fato gere desconfiança na consultoria e fracasso do projeto de melhoria".

- Estratégias e políticas organizacionais: As propriedades deste conceito apontam para questões críticas como políticas de reconhecimento à colaboração na melhoria dos processos, conflitos de interesses na implementação de processos e 
programas de incentivo para capacitação e crescimento profissional dos membros da organização. Essas questões foram identificadas como causadoras de problemas que afetam o sucesso de iniciativas de melhoria como rotatividade de pessoal e falta de competências em engenharia de software dos membros da organização.

- Programa de Melhoria de Processos: Foram identificadas 8 propriedades associadas a esse conceito, indicativas de aspectos críticos relacionados ao gerenciamento da iniciativa de melhoria, como disponibilidade de recursos financeiros e humanos, adequação do planejamento e envolvimento dos membros da organização na iniciativa de melhoria e tratamento da iniciativa como um projeto real na organização. Segundo os gerentes e os membros de equipes de implementação "quando a iniciativa de melhoria não é tratada como um projeto real na organização, é mais provável ter-se dificuldade com o projeto de melhoria".

- Ambiente: As características ambientais críticas para ter sucesso na condução de iniciativa de melhoria estão relacionadas a 6 propriedades variando desde a adequação da estrutura e estabilidade interna da organização até a existência de eventos internos ou externos capazes de afetar a organização e a constância do fluxo de projetos. Essas características afetam de forma crítica o programa de melhoria, pois têm impacto direto na disponibilidade de recursos financeiros da organização para atividades de melhoria de processo. Segundo o coordenador da instituição de consultoria "quando existem problemas externos que influenciam a saúde da empresa ou o fluxo de projetos, é provável ter-se problemas de instabilidade interna na organização".

- Processos de software: O processo de software é o conceito central de qualquer iniciativa de melhoria e, conseqüentemente, exerce influência crítica no seu sucesso. Os dados analisados permitiram identificar 5 propriedades relacionadas a este conceito que apontam basicamente para questões de adequação e alinhamento da definição dos processos para as especificidades e os objetivos estratégicos da organização, além de outras questões relacionadas ao uso dos processos, como completa institucionalização das melhorias implementadas nos projetos e adequação do planejamento da execução dos processos nos projetos.

- Recursos de software e hardware de apoio aos processos de software: Foram identificadas 3 propriedades deste conceito relacionadas à adequação das ferramentas de apoio, disponibilidade de recursos de software e hardware de apoio à execução dos processos e adequação do processo de seleção das ferramentas de apoio. Segundo o coordenador da instituição de consultoria "quando a empresa não tem ferramentas de apoio é mais difícil a implementação de processos e a implantação de melhorias".

\section{Comportamento Estratégico na Implementação de Melhorias em Processos de Software}

A segunda categoria central da teoria é constituída de conceitos e seus respectivos atributos que representam o processo de mudança organizacional do ponto de vista da organização de consultoria e que descrevem a interação entre os atores da iniciativa de 
melhoria e o contexto institucional no qual melhorias são implementadas. É importante destacar que este processo e as ações associadas não cobrem completamente as atividades necessárias para executar uma iniciativa de melhoria, mas expressam as ações estratégicas críticas que devem ter atenção especial para maximizar as chances de obtenção de sucesso. Este processo é dividido em 5 subprocessos descritos a seguir:

- Iniciando o projeto de consultoria em melhoria de processos: Este subprocesso engloba tipos de ação consideradas relevantes de serem tomadas no início do projeto de consultoria para subsidiar a tomada de decisão das metas a serem alcançadas. Essas ações visam entender o contexto da iniciativa de melhoria em termos do perfil dos membros da organização (sexo, idade etc), além de outras ações para entender as características da organização alvo das melhorias que podem ter influência no sucesso da iniciativa de melhoria, como a saúde financeira da empresa e o fluxo de projetos previsto para o período de consultoria. Segundo o coordenador da instituição de consultoria "fazer um diagnóstico da empresa, da forma de trabalho e do conhecimento e competências da equipe para ter subsídios mais seguros para a tomada de decisão do nível possível de ser alcançado e do prazo para isto, ajuda a diretoria a acatar a sugestão da consultoria sobre o nível e cronograma do projeto de melhoria".

- Planejando o projeto de consultoria em melhoria de processos: Este subprocesso engloba tipos de ação relacionadas à definição da estratégia de definição de processos de software e à definição da estratégia de introdução de mudanças nos processos, bem como à seleção dos projetos para a iniciativa de melhoria e à definição dos participantes da iniciativa de melhoria e suas responsabilidades, além de outras ações relacionadas à seleção das ferramentas de apoio aos processos de software. A execução dessas ações no estágio de planejamento da iniciativa de melhoria permite antever e facilitar o tratamento de potenciais problemas capazes de afetar o sucesso da iniciativa.

- Implantando melhorias nos processos de software: Os tipos de ação que compõem este subprocesso estão diretamente relacionados a aspectos práticos para implementação de melhorias, como a recomendação de mudanças nas estratégias e políticas organizacionais, além de recomendação da adoção de mecanismos para gerenciamento dos recursos humanos e realização de treinamentos dos membros da organização envolvidos na iniciativa de melhoria. Um tipo de ação que se destaca neste subprocesso pelo seu alto grau de densidade está relacionado a ações de conscientização da alta direção e dos membros da organização para melhoria de processos. Este tipo de ação garante ou maximiza, bem como elimina ou minimiza uma grande quantidade de características de contexto que podem influenciar o sucesso de iniciativas de melhoria, como a falta de motivação ou dificuldade para aceitação de mudanças. Segundo o coordenador da instituição de consultoria "explicar os potenciais benefícios para a carreira de cada colaborador e como a melhoria de processos vai ajudar a melhorar o trabalho na empresa e organizar o trabalho de cada um pode ajudar a superar o problema da falta de interesse ou motivação dos membros da organização pelo projeto de melhoria".

- Acompanhando e controlando a implantação das melhorias nos processos: Este subprocesso engloba tipos de ação relevantes para aumentar a visibilidade dos 
resultados obtidos com a implementação de melhorias em processos como a avaliação interna da implantação ao final de cada projeto de software, bem como ações de comunicação dos resultados alcançados com a implantação das melhorias nos processos tanto quantitativos quanto qualitativos da própria organização ou de outras organizações. Também faz parte deste subprocesso ações de replanejamento do projeto de consultoria em melhoria de processos para tratar problemas e desvios identificados no acompanhamento da implantação e que podem envolver desde o replanejamento do cronograma e freqüência de apoio da consultoria até abortar a iniciativa de melhoria. Segundo o coordenador da instituição de consultoria "caso se perceba um grave problema de fluxo de projetos e a empresa não seja participante de grupo com apoio SOFTEX, pode ser mais adequado abortar o projeto de melhoria".

- Gerenciando os recursos humanos da consultoria especializada em melhoria de processos: Como este estudo teve como propósito investigar iniciativas de melhorias no ponto de vista de uma organização de consultoria, foram observados tipos de ações que devem ser tomadas internamente à organização de consultoria para ter sucesso na condução de iniciativas de melhoria. Essas ações estão relacionadas à capacitação dos membros da consultoria para desempenhar atividades nos projetos de consultoria, bem como ações para garantir uma seleção adequada dos consultores e desta forma evitar a ocorrência de problemas pela falta de adequação do perfil dos consultores alocados nas iniciativas de melhoria. Segundo o coordenador da instituição de consultoria "para garantir conhecimento, habilidades e experiência à sua equipe é importante que a consultoria possua uma política de treinamento e formação continuada".

\section{Conclusão}

Este trabalho apresentou os resultados da aplicação da Grounded Theory para apoiar a construção de uma teoria baseada nas experiências individuais de consultores de melhorias em processos. Por meio da Grounded Theory, foi possível desenvolver um entendimento do significado, experiências, eventos e realidade do fenômeno associado ao sucesso de iniciativas de implementação de melhorias em processos de software.

Neste trabalho, foi demonstrada a aplicabilidade de Grounded Theory para apoiar investigações sobre implementação de melhorias em processos. Além disso, foi desenvolvido um framework teórico capaz de explicar: (i) os fatores de contexto que exercem tanto influência positiva quanto negativa no sucesso de iniciativas de melhoria e (ii) as ações representando a interação entre os principais atores do fenômeno de implementação de melhorias e o contexto institucional para ter sucesso na condução de iniciativas de implementação de melhorias em processos de software.

No estágio atual deste trabalho, está sendo conduzida a avaliação da teoria por coordenadores de diferentes organizações de consultoria. Os resultados dessa avaliação serão posteriormente analisados com o propósito de identificar questões que devem ser investigadas para evoluir a teoria. Isto permitirá obter um entendimento mais preciso do fenômeno de implementação de melhorias em processos de software. 


\section{Referências}

Baddoo, N., Hall, T., 2003, "De-motivators for software process improvement: An analysis of practitioners' views", Journal of Systems and Software, v. 66, n. 1, pp. 23-33.

Bandeira-De-Mello, R., Cunha, C., 2003, "Operacionalizando o método da Grounded Theory nas Pesquisas em Estratégia: técnicas e procedimentos de análise com apoio do software ATLAS/TI", Curitiba, Brazil.

Carvalho, L., Scott, L., Jeffery, R., 2003, Exploring the use of Techniques from Grounded Theory in Process Engineering, 03/1, Centre for Advanced Software Engineering Research (CAESER), Sydney, Australia.

Chrissis, M.B., Konrad, M., Shrum, S., 2006, CMMI: Guidelines for Process Integration and Product Improvement, 2nd ed., Addison-Wesley.

Coleman, G., O'connor, R., 2007, "Using grounded theory to understand software process improvement: A study of Irish software product companies", Information and Software Technology, v. 49, n. 6, pp. 654-667.

Coleman, G., O'connor, R., 2008, "Investigating software process in practice: A grounded theory perspective", Journal of Systems and Software, v. 81, n. 5, pp. 772-784.

Montoni, M., Rocha, A.R., 2007, "A Methodology for Identifying Critical Success Factors that Influence Software Process Improvement Initiatives: An Application in the Brazilian Software Industry", Lecture Notes in Computer Science (LNCS), LNCS 4764, EuroSPI - European Systems \& Software Process Improvement and Innovation (Setembro), pp. 175-186.

Orlikowski, W.J., 1993, "CASE Tools as Organizational Change: Investigating Incremental and Radical Changes in Systems Development", Management Information Systems Quarterly, v. 17, n. 3.

Seaman, C.B., 1999, "Qualitative methods in empirical studies of software engineering", IEEE Transactions on Software Engineering, v. 25, n. 4, pp. 557572.

Softex, 2009, "MPS.BR - Melhoria de Processo do Software Brasileiro, Guia Geral (v. 2009)". In: http://www.softex.br/mpsbr/_guias/default.asp.

Staples, M., Niazi, M., Jeffery, R., et al., 2007, "An exploratory study of why organizations do not adopt CMMI", Journal of Systems and Software, v. 80, n. 6, pp. 883-895.

Strauss, A., Corbin, J.M., 1998, Basics of Qualitative Research: Techniques and Procedures for Developing Grounded Theory, 2nd ed., Sage Publications. 